\title{
An image segmentation framework for extracting tumors from breast magnetic resonance images
}

\author{
Le Sun*, , Jinyuan $\mathrm{He}^{\dagger}$, Xiaoxia Yin ${ }^{\dagger}$, Yanchun Zhang ${ }^{\dagger}$, Jeon-Hor Chen ${ }^{\ddagger}$, , \\ Tomas Kron ${ }^{\top}$ and Min-Ying $\mathrm{Su}^{*}$ \\ *School of Computer and Software \\ Nanjing University of Information Science \\ and Technology, P. R. China \\ ${ }^{\dagger}$ Center for Applied Informatics \\ Victoria University, Australia \\ *enter for Functional Onco-Imaging of the \\ Department of Radiological Sciences \\ University of California Irvine, USA \\ $\S^{\S}$ Department of Radiology \\ E-Da Hospital and I-Shou University, Kaohsiung, Taiwan \\ "Peter MacCallum Cancer Centre, Australia \\ "sunle2009@gmail.com
}

Received 5 December 2017

Accepted 14 January 2018

Published 9 March 2018

\begin{abstract}
Magnetic resonance imaging (MRI) has been a prevalence technique for breast cancer diagnosis. Computer-aided detection and segmentation of lesions from MRIs plays a vital role for the MRIbased disease analysis. There are two main issues of the existing breast lesion segmentation techniques: requiring manual delineation of Regions of Interests (ROIs) as a step of initialization; and requiring a large amount of labeled images for model construction or parameter learning, while in real clinical or experimental settings, it is highly challenging to get sufficient labeled MRIs. To resolve these issues, this work proposes a semi-supervised method for breast tumor segmentation based on super voxel strategies. After image segmentation with advanced cluster techniques, we take a supervised learning step to classify the tumor and nontumor patches in order to automatically locate the tumor regions in an MRI. To obtain the optimal performance of tumor extraction, we take extensive experiments to learn parameters for tumor segmentation and classification, and design 225 classifiers corresponding to different parameter settings. We call the proposed method as Semi-supervised Tumor Segmentation (SSTS), and apply it to both mass
\end{abstract}

${ }$ Corresponding author.

This is an Open Access article published by World Scientific Publishing Company. It is distributed under the terms of the Creative Commons Attribution 4.0 (CC-BY) License. Further distribution of this work is permitted, provided the original work is properly cited. 
and nonmass lesions. Experimental results show better performance of SSTS compared with five state-of-the-art methods.

Keywords: Breast lesion; image segmentation; MRI.

\section{Introduction}

Image segmentation is a process of separating a digital image into different regions for specific purposes, such as object recognition and classification. ${ }^{1}$ It is widely used in the area of medical imaging analysis to precisely and automatically delineate Regions of Interests (ROIs). Computer-aided image segmentation is a key task in intelligent biomedical image processing applications, like disease diagnosis, anatomical structure analysis, and pathology localization. $^{2}$

Breast cancer is one of the most common cancers that causes deaths in women worldwide. ${ }^{3}$ Magnetic resonance imaging (MRI) is an advantageous technique for breast cancer diagnosis. Radiologists assess MRIs layer-by-layer to locate lesions and diagnose diseases, which is an arduous and timeconsuming task in real clinical applications. ${ }^{4}$ Therefore, computer-aided detection and segmentation of lesions from MRIs plays a vital role for the MRI-based disease analysis.

Numbers of researchers investigated the problem of lesion segmentation in MRIs in past decades. ${ }^{5}$ Specifically, Fuzzy C means (FCM) clustering is one of the most prevailing methods of breast tumor segmentation due to its simplicity. ${ }^{4}$ Markov random field (MRF)-based methods have also been explored for lesion and anatomical structure segmentation. ${ }^{6}$ In addition, Liney et $a .^{7}$ and Chen et al. ${ }^{8}$ adopted region growing techniques to outline lesions.

The main weaknesses of the existing breast lesion segmentation include the following aspects: (1) some methods require manual delineation of ROIs as a step of initialization (e.g., Ref. 9), which restricts the automatic segmentation to cases that sufficient expert knowledge should be known in advance ${ }^{6}$; (2) supervised methods require a large amount of labeled images for model construction or parameter learning, while in real clinical or experimental settings, it is highly challenging to get sufficient labeled MRIs due to the limited number of patients and the time constraint; (3) existing work mainly focuses on segmenting mass tumors (either benign or malignant) (e.g., Ref. 5), the investigation on segmenting nonmass lesions however is relatively less due to the shape diversity of the lesions, which makes the delineation of the lesions extremely difficult.

To tackle these issues, we propose a Semisupervised Tumor Segmentation (SSTS) technique, which constraints the number of threshold parameters that need to be set in advance, and requires a small amount of labeled images. In addition, we take a supervised classification step to classify the tumor and nontumor patches obtained from the unsupervised segmentation to automatically locate the tumor regions in an MRI. SSTS comprises four main steps: at first, we delineate an approximate area of tumors in an MRI (namely approximate area), by segmenting an MRI based on Otsu's thresholding method ${ }^{10}$ with respect to pixel intensity levels and removing the clusters in low intensity levels, as tumors in MRI images normally have relatively high intensities. Secondly, oversegment the approximate area based on the Simple Linear Iterative Clustering (SLIC) method $^{11}$ to form super-pixels, which further reduces the number of pixels and improves the clustering efficiency in the sequel stages. Thirdly, group super-pixels based on the DBSCAN technique ${ }^{12}$ in terms of mean intensities and positions of super-pixels. Finally, classify tumor patches to locate tumors in the original image by using an Adaboost-M1 classification algorithm ${ }^{13}$ based on 20 texture features ${ }^{9}$ and mean intensity levels of patches.

To obtain the optimal performance of tumor extraction, we take extensive experiments for tumor segmentation and classification, with 225 classifiers to be designed corresponding to different parameter settings.

The main contributions of this paper are: (1) we design a semi-supervised method for extracting breast tumors in MRIs, which requires smaller labeled datasets compared with the full supervised methods; (2) we apply the proposed method to both mass and nonmass lesions and present the tumor extraction performance of both of them and (3) abundant experimental results show better performance of our work compared with five state-ofthe-art image segmentation methods, e.g., a multithreshold (MT) method ${ }^{10}$ and an FCM method. ${ }^{14}$ 
This paper is structured as follows: Sec. 2 illustrates the related work of tumor segmentation; Sec. 3 introduces the proposed SSTS framework in detail; Sec. 4 discusses the experimental settings and results and Sec. 5 concludes this paper and indicates our future work.

\section{Related Work}

Unsupervised learning methods are normally used for the identification of tumors. ${ }^{15}$ In the research work carried out by Sauwen et al., ${ }^{16}$ the performance of unsupervised classification algorithms is illustrated, for the segmentation of high-grade gliomas based on multi-parametric MRI modalities including conventional MRI, diffusion-weighted imaging, magnetic resonance spectroscopic imaging, and perfusion-weighted imaging. Pereira et al. ${ }^{17}$ applied Convolutional Neural Network (CNN) with small $3 \times 3$ kernels to automatically segment brain tumors from MRI images. The authors employed the intensity normalization as a preprocessing for CNN-based segmentation.

Unsupervised methods like FCM and Particle Swarm Optimization (PSO) have been applied to brain tumor segmentation. Sehgal et al. ${ }^{18}$ proposed an automatic method to segment brain tumors from MRIs. The method include five steps: Image Acquisition, Preprocessing, Segmentation using FCM technique, Tumor Extraction and Evaluation. Al-Faris et al. ${ }^{19}$ proposed an unsupervised approach for MRI tumor segmentation by combining Seeded Region Growing (SRG) and PSO.

In addition, Chen et $a l_{.}{ }^{4}$ proposed an FCM clustering-based method to segment breast lesions from the three-dimensional contrast-enhanced MR images. The drawback of this method is its requirement of manually drawing ROIs as an initialization step. The authors of Ref. 6 developed a multi-channel MRFs framework, in which they use conditional mutual information to search for conditional independent features. This method requires a large amount of labeled data as a priori for model construction, and it does not analyze the segmentation results on nonmass tumors by using the proposed method. Yin et $a .^{20}$ proposed a new methodology based on tensor algebra that uses a higher order singular value decomposition to perform three-dimensional voxel reconstruction from a series of temporal images obtained using dynamic contrast-enhanced MRI (DCE-MRI). Principal component analysis (PCA) is used to robustly extract the spatial and temporal image features and simultaneously denoise the datasets. This method, however, is validated for tumor segmentation with small size.

\section{SSTS Framework Description}

Figure 1 shows a flowchart of the SSTS technique. A preprocessing module is proposed to normalize MRIs after breast separation from chests using simple image processing operation. As shown in Fig. 1, Module1 delineates the approximate area based on the Otsu thresholding of imaged intensity. As imaged tumors in MRIs normally show high intensity levels, the clustered pixels with low intensity are removed. The approximate area is over-segmented to superpixels using SLIC (Module2). Our work has empirically shown that the thresholding step can improve the efficiency of the over-segmentation by removing low intensity noise. Module3 clusters super-pixels based on the DBSCAN technique in terms of mean intensities and positions. As a lesion is normally presented as a connected area in an MRI, this step groups super-pixels with similar intensity, and meanwhile are spatially adjacent (according to 8adjacency matrix) to each other.

After DBSCAN clustering (Module4), we get a set of tumor and nontumor patches, where a (non)tumor patch means the part of a (non) tumor area or the part that covers a (non) tumor area (see Fig. 1). To achieve a supervised learning operation, we define a labeled patch as follows: if the area of a patch coinciding with the ground truth tumor area is above a threshold $(r)$, it is a tumor patch. We use the dice ratio (DR) between a patch and a ground truth to measure the coinciding area of tumor and nontumor patches.

Each labeled patch (tumor/nontumor) is described by 21 features (20 texture features and mean intensity). The features of each patch are stored in the Patch database. An Adaboost classifier is trained for patch classification based on the labeled patches and their features in Patch database. The classified patches are combined together to form a tumor area in an MRI.

\subsection{Delineate approximate tumor area based on Otsu thresholding}

The first step of SSTS is to delineate an approximate area completely covering tumors (namely 


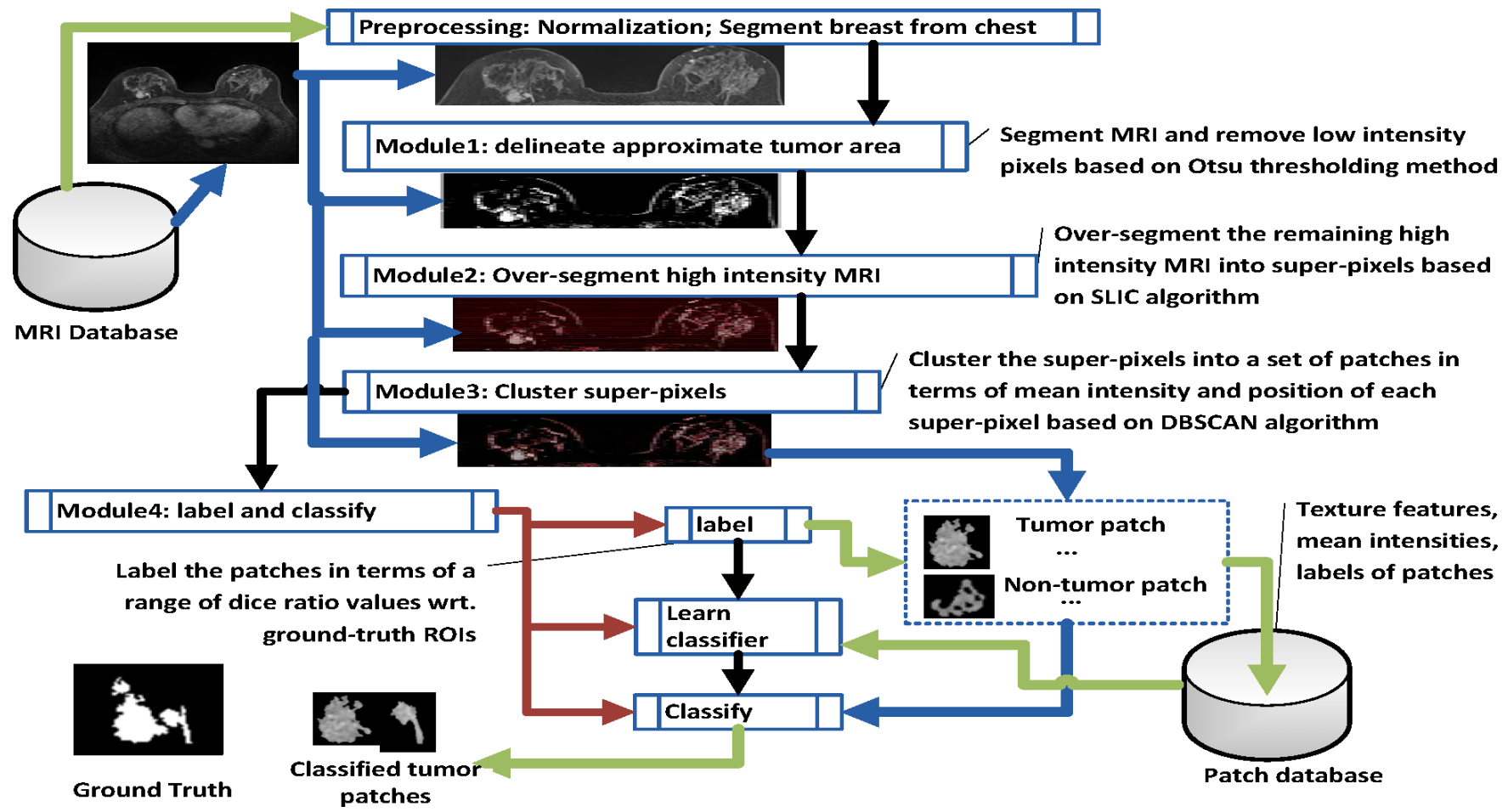

Fig. 1. Flowchart of SSTS framework.

approximate area) based on Otsu's thresholding method. The sequel steps will be only based on the approximate area.

The Otsu's method aims to finding an optimal threshold value $(k)$ separating pixels of a gray-level image $I$ into two classes: foreground $\left(C_{\mathrm{fg}}\right)$ and background $\left(C_{\mathrm{bg}}\right)$. It is implemented by minimizing the weighted within-class variance $\left(\sigma_{\text {in }}\right)^{2}$ or maximizing the between-class variance $\left(\sigma_{\mathrm{bt}}^{2}\right)$. This threshold finding process is based on histograms of the gray-level image. Assume $I$ has $L$ intensity levels, $N$ pixels, and the number of pixels $N$ at level $i$ is represented as $n_{i}(i \in[1, L])$, then the histogram $p_{i}$ of $I$ is normalized as follows:

$$
p_{i}=n_{i} / N, \quad p_{i} \geq 0, \quad \sum_{i=1}^{L} p_{i}=1 .
$$

Suppose $C_{\mathrm{bg}}$ of image $I$ contain pixels at levels $[1, \ldots, k], C_{\mathrm{fg}}$ contain pixels at levels $[k+1, \ldots, L]$, $\omega(k)$ and $\mu(k)$ be the zeroth- and the first-order cumulative moments of the histogram up to the $k$ th level, respectively, then the occurrence probabilities $\left(\omega_{\mathrm{fg}}\right.$ and $\left.\omega_{\mathrm{bg}}\right)$ and mean intensities $\left(\mu_{\mathrm{fg}}\right.$ and $\left.\mu_{\mathrm{bg}}\right)$ are

$$
\omega_{\mathrm{bg}}=\operatorname{Pr}\left(C_{\mathrm{bg}}\right)=\sum_{i=0}^{k} p_{i},
$$

$$
\begin{gathered}
\omega_{\mathrm{fg}}=\operatorname{Pr}\left(C_{\mathrm{fg}}\right)=\sum_{i=k+1}^{L} p_{i}, \\
\mu_{\mathrm{bg}}=\sum_{i=k+1}^{L} i \operatorname{Pr}\left(i \mid C_{\mathrm{bg}}\right)=\sum_{i=k+1}^{L} i p_{i} / \omega_{\mathrm{bg}}=\mu(k) / \omega(k), \\
\mu_{\mathrm{fg}}=\sum_{i=1}^{k} i \operatorname{Pr}\left(i \mid C_{\mathrm{fg}}\right)=\sum_{i=1}^{k} i p_{i} / \omega_{f g}=\frac{\mu(L)-\mu(k)}{1-\omega(k)} .
\end{gathered}
$$

The objective is to find a $k^{*}$ that maximizes $\sigma_{\mathrm{bt}}^{2}$ :

$$
\max \left(\sigma_{\mathrm{bt}}^{2}\right)=\frac{\left[\mu(L) \omega\left(k^{*}\right)-\mu\left(k^{*}\right)\right]^{2}}{\omega\left(k^{*}\right)\left[1-\omega\left(k^{*}\right)\right]} .
$$

Pixels of image $I$ are then separated into $C_{\mathrm{fg}}$ and $C_{\mathrm{bg}}$ based on $k^{*}$. Figure 2 shows examples of the segmentation results of Otsu's method on both mass and nonmass MRIs.

The sequel tasks of SSTS will only work on the foreground image (i.e., $C_{\mathrm{fg}}$ ), as tumors in an MRI are normally with high intensity levels. The background part is removed to improve the efficiency of tumor extraction. 


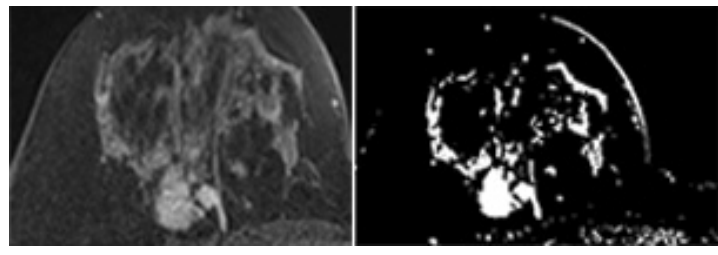

(a) case 1

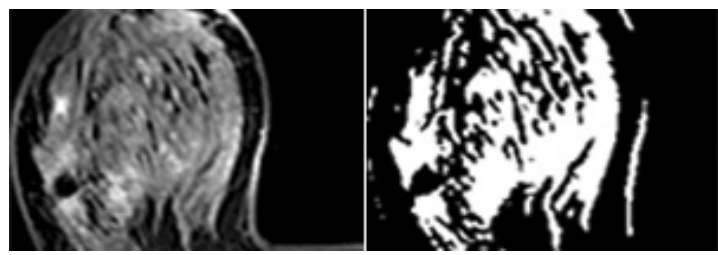

(c) case 7

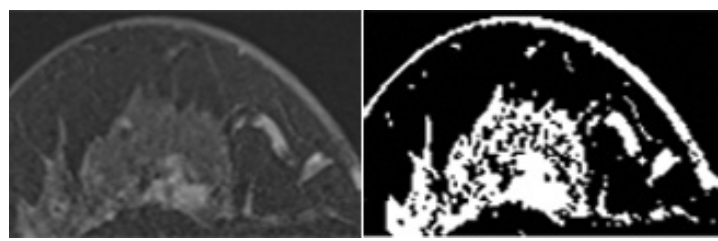

(e) case 18

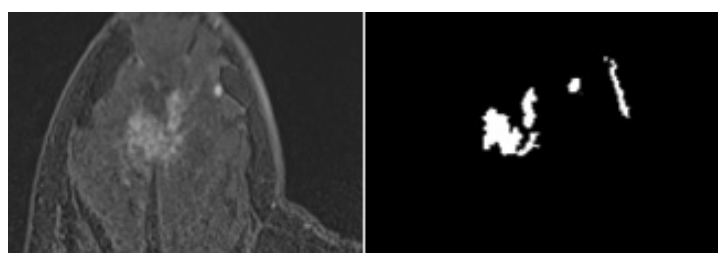

(b) case 6

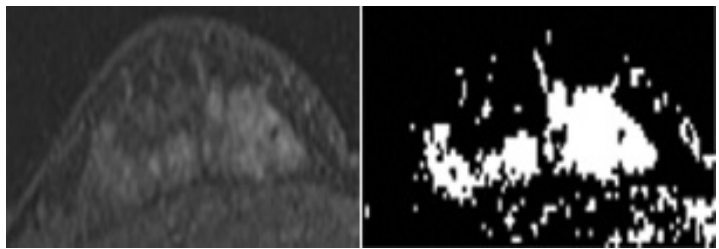

(d) case 16

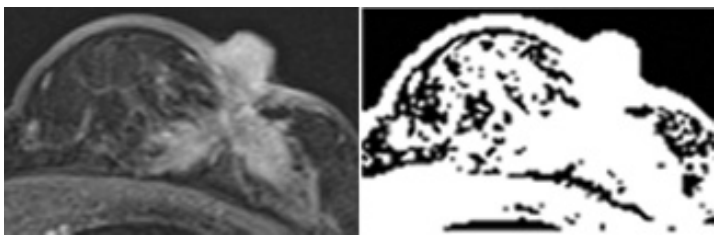

(f) case 20

Fig. 2. Foreground of MRIs containing both mass (cases 1,6 and 7) and nonmass (cases 16, 18 and 20) tumors, segmented based on Otsu's method.

\section{2. $\quad$ Segment MRIs into super-pixels based on SLIC}

We use SLIC algorithm to cluster image pixels into super-pixels. SLIC contains four steps: initialization, assignment, update and post-processing. Suppose image $I$ has $N$ pixels, and $k$ is the number of super-pixels that $I$ is going to be segmented. The initialization step initializes centers of super-pixel clusters $C_{i}=\left(l_{i}, s_{i}\right)$, where $l_{i}$ is the intensity level of center of the $i$ th cluster, and $s_{i}$ is the location $\left(x_{i}, y_{i}\right)$ of the center pixel. The grid interval of super-pixel clustering is set as $g=\sqrt{(N / k)}$ to produce equally-sized search regions to speed up forming similar-sized super-pixels. The centers are initialized as the positions with lowest gradients in a $3 \times 3$ neighborhood to reduce the chance that a center is located on an edge or a noisy pixel.

The assignment step assigns each pixel to a region-overlapped cluster. The search region of clustering a pixel is limited to an area, which speeds up the formation of super-pixels, as limited search regions reduce the times of distance calculation between pixels and clustering centers. SLIC employs a new distance calculation method
(Eq. (7)) to achieve pixel clustering based on both pixel intensity levels and locations:

$$
D=\sqrt{d_{i}^{2}+\left(\frac{d_{s}}{S}\right)^{2} m^{2}},
$$

where $d_{i}$ and $d_{s}$ are the intensity and location distances between two pixels, respectively; and $m$ is a constant that normalizes intensity distances, which is in the range of $[1,40]$.

The update step changes each cluster center $\left(C_{i}\right)$ to the mean intensity and location vectors $\left\langle\mu\left(l_{i}\right)\right.$, $\left.\mu\left(s_{i}\right)\right\rangle$ of all pixels in each cluster. The final superpixels are determined when the residual error between the previous cluster center locations and the updated cluster center locations converges. Based on the empirical validating, the residual error can converge after 10 iterations. At last, the post-processing step assigns the remaining disjoint pixels to nearby super-pixels in terms of locations.

Examples of super-pixel segmentation are shown in the left part of each sub-figures in Fig. 3 for oversegmenting six MRIs containing mass tumors, and in Fig. 4 for nonmass tumors. 

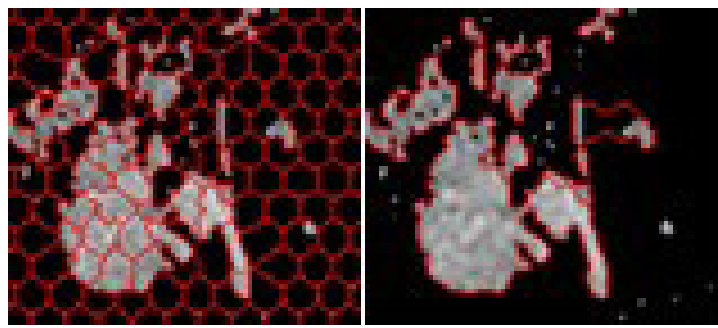

(a) case 3

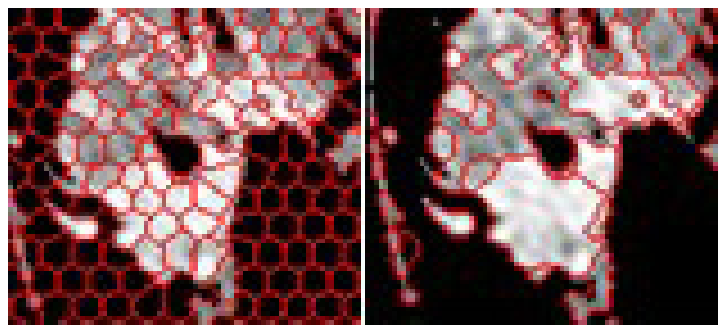

(c) case 7

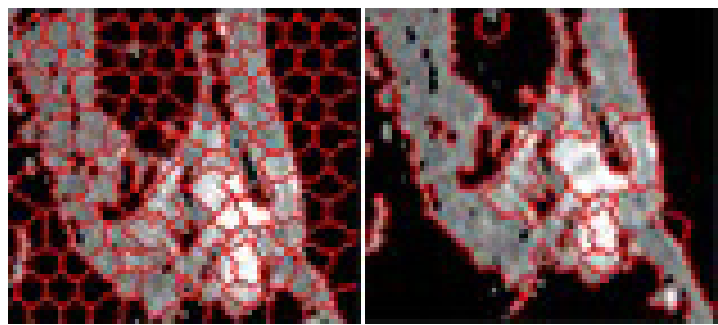

(e) case 9

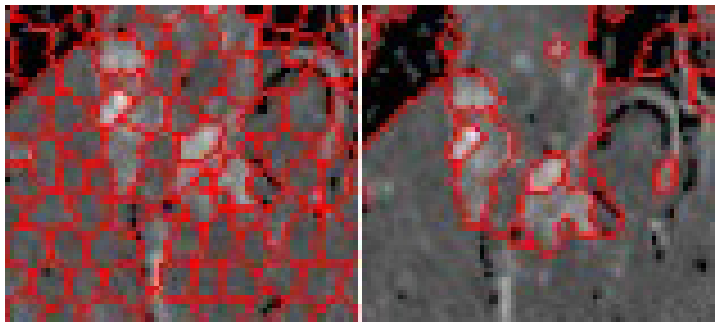

(b) case 6

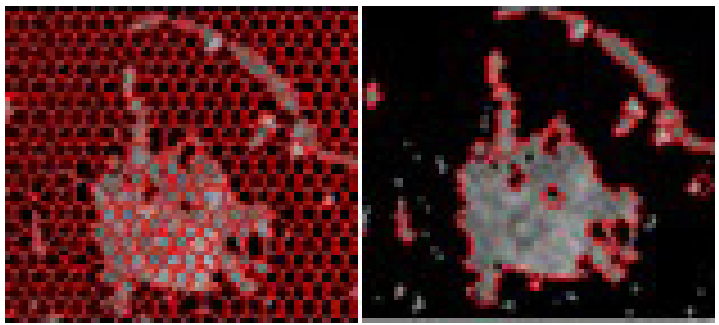

(d) case 8

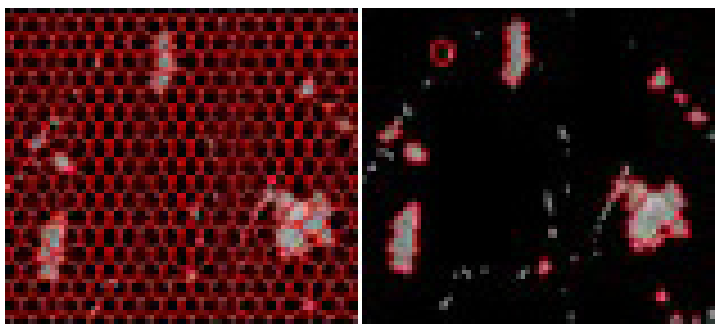

(f) case 14

Fig. 3. SLIC (left) and DBSCAN (right) segmentation of six mass tumors based on $m=15$ and dist $=8$.

\subsection{Cluster super-pixels into patches based on DBSCAN technique}

After over-segmentation of MRIs in Module 2, Module 3 clusters super-pixels to form tumor and nontumor patches based on DBSCAN technique, which is processed as follows:

Given $O$ as a dataset of $N$ items, $o_{i} \in O$, then $\operatorname{den}\left(o_{i}\right)$ represents the number of neighbors in the neighborhood of the $i$ th items. The neighbor of $o_{i}$ is defined as an element $o_{j}$ that $\operatorname{dist}\left(o_{i}, o_{j}\right)<\varepsilon$ and $\mathrm{NB}_{\varepsilon}\left(o_{i}\right)$ is the set of all neighbors of $i$.

There are three types of objects distinguished by the number of neighbors of an object: core, border, and noise. Assume $\min _{\mathrm{NB}}$ is the minimum number of neighbors in the neighborhood of object $o_{i}$, i.e., $\operatorname{den}\left(o_{i}\right) \geq \min _{\mathrm{NB}}$, then $o_{i}$ is called a core object, denoted by $o_{i}^{\text {core }}$. If the number of neighbors of an object $o_{j}$ is less than $\min _{\mathrm{NB}}$, i.e., $\operatorname{den}\left(o_{j}\right)<\min _{\mathrm{NB}}$, and $o_{j} \in \mathrm{NB}_{o_{i}^{\text {core }}}$, then $o_{j}$ is called border object, denoted by $o_{j}^{\text {border }}$. A noise object, $o^{\text {noise }}$, has less than $\min _{\mathrm{NB}}$ neighbors in the range of distance $\varepsilon$, and all the neighbors are not core objects, i.e., den $\left(o^{\text {noise }}<\min _{\mathrm{NB}}\right)$ and $\forall o_{j} \in \mathrm{NB}_{\varepsilon}\left(o_{i}\right), o_{j}$ is not $o_{j}^{\text {core }}$.

If an object $o_{i}$ is density reachable with object $o_{j}, i \geq 1, j \geq 2$, then there is a directed path $o_{i}, \ldots$, $o_{r}, \ldots, o_{j}$, and $\forall o_{r}, \operatorname{den}\left(o_{r}\right) \geq \min _{\mathrm{NB}}, o_{r+1} \in \mathrm{NB}_{\varepsilon}\left(o_{r}\right)$. An object in a cluster is density-reachable from arbitrary core objects in the cluster.

The DBSCAN clustering process has two steps: arbitrarily identify a core object $o_{1}$, and then recursively recognize the density-reachable objects of $o_{1}$ to form a cluster. In the DBSCAN clustering of super-pixels, we use 8-connected adjacency matrix ${ }^{21}$ to record the adjacent relation among super-pixels. If two super-pixels are adjacent with each other, their distance is the mean pixel intensity distance; if they are not adjacent, their distance is infinite. ${ }^{22}$ 

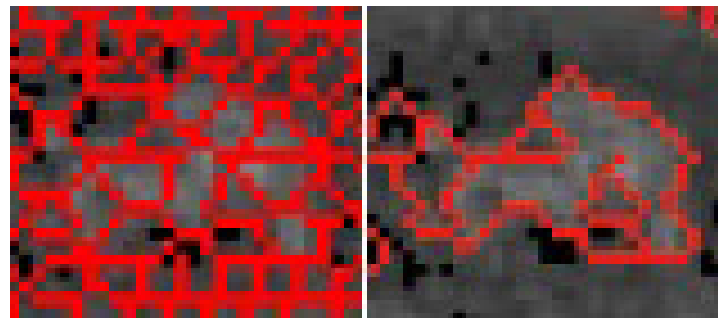

(a) case 16
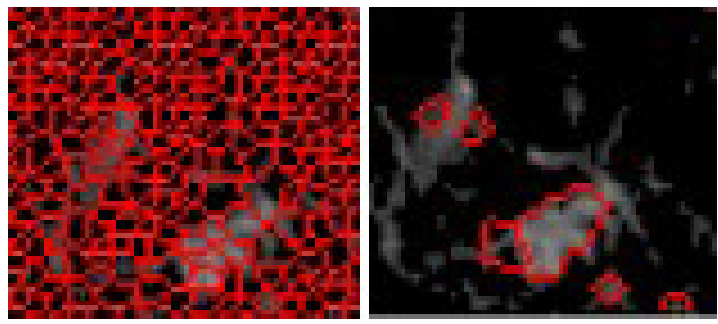

(c) case 18

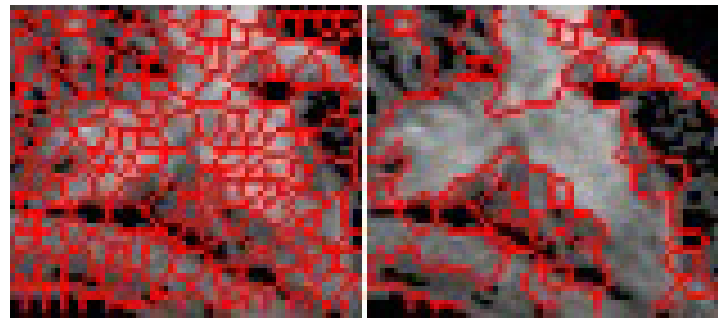

(e) case 20

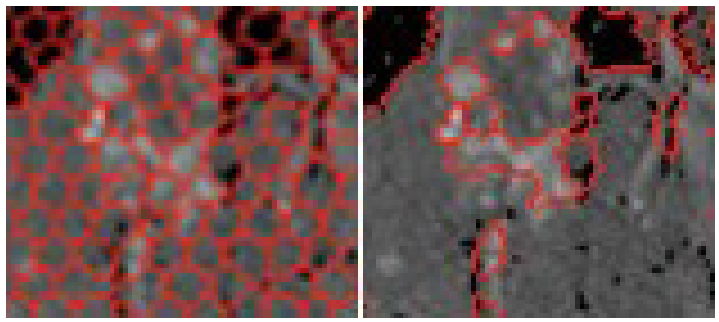

(b) case 17

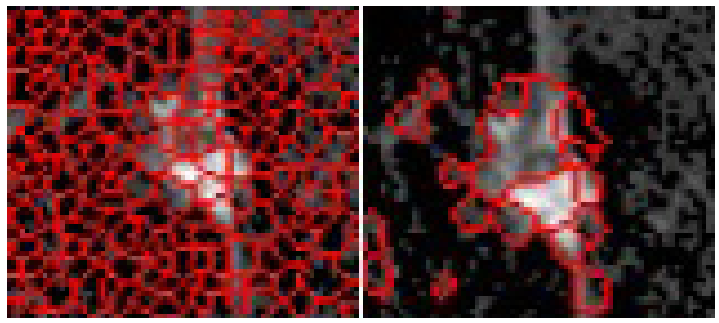

(d) case 19

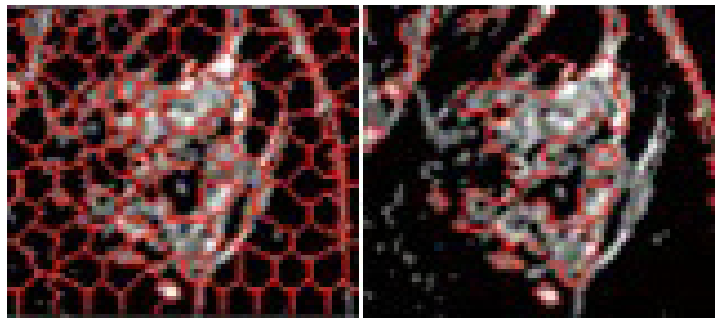

(f) case 21

Fig. 4. SLIC (left) and DBSCAN (right) segmentation of six nonmass tumors based on $m=15$ and dist $=8$.

The results of DBSCAN clustering of mass tumors of MRIs are shown in the right part of Figs. 3(a)-3(f). And the tumor segmentation of six nonmass tumors of MRIs are shown in the right part of Figs. 4(a)-4(f). DBSCAN classifies superpixels that are close to each other in terms of both intensities and spatial distances. The over-segmented patches by DBSCAN will be classified by Adaboost-M1 algorithm in the next stage to locate the patches containing tumors.

\subsection{Classify image patches to extract tumors based on Adaboost-M1}

After segmenting an MRI into a set of patches, we take a classification step to predict the patches belonging to the tumor part in the MRI. Boosting algorithms ${ }^{23}$ are used to improve the performance of weak classifiers. The performance of a weak classifier is normally only better than random classification. We denote a weak classifier as a map $f$ : $X \rightarrow Y$, where $x \in X$ is a feature vector of an object that needs to be classified, $y \in Y$ is a label indicating the class of $x$, and $|Y| \geq 2$. At iteration $t$, the boosting algorithm gives the weak learner a distribution $\left(D_{t}\right)$ on a training set $R=$ $\left\{\left(x_{1}, y_{1}\right), \ldots,\left(x_{n}, y_{n}\right)\right\}$, and the weak learner then trains a classifier $h_{t}: X \rightarrow Y$ that minimizes the training error $\varepsilon=\sum_{i} D_{t}\left(x_{i}\right) \delta\left(h_{t}\left(x_{i}\right) \neq y_{i}\right)$, where if $h_{t}\left(x_{i}\right) \neq y i, \delta=1$; or else, $\delta=0$. This error minimization procedure is conducted in $T$ iterations so that classifiers $h_{1}, \ldots, h_{T}$ are trained, and then the boosting algorithm calculates the final classifier $h_{f}$ based on the $T$ classifiers.

Adaboost assists the weak learner becoming stronger by focusing on the objects that are hard to be classified. That is, in each iteration of weight updates $\left(D_{t} \rightarrow D_{t+1}\right)$, the boosting algorithm 
assigns higher weights to the misclassified objects in round $t$ by multiplying a number in $[0,1)$ with the weights of objects that are correctly classified. $D$ is updated as follows:

$$
D_{t+1}\left(x_{i}, y\right)=\frac{D_{t}\left(x_{i}, y\right)}{Z_{t}} \cdot \beta_{t}^{\frac{1}{2}\left(1+h_{t}\left(x_{i}, y_{i}\right)-h_{t}\left(x_{i}, y\right)\right)},
$$

where $\beta_{t}=\frac{\varepsilon_{t}}{1-\varepsilon_{t}}$, and $Z_{t}$ is a normalization constant.

The final classifier is calculated as follows:

$$
h_{f}\left(x_{i}\right)=\arg \max \sum_{t=1}^{T}\left(\ln \frac{1}{\beta_{t}}\right) h_{t}\left(x_{i}, y_{i}\right),
$$

where the multiplication of $\ln \frac{1}{\beta_{t}}$ makes the higher weights be assigned to weak classifiers having better performance.

We use 20 texture features and mean intensity level of a patch to classify tumor and nontumor patches based on Adaboost-M1 algorithm. There are several ways for describing image textures,${ }^{24}$ like the two-dimensional Fourier transformation and the gray-level co-occurrence metrics (GLCM). The Fourier transform mainly reveals the periodicity and directionality of an image, ${ }^{31}$ while $\mathrm{GLCM}^{25}$ is a more powerful and popular texture description method that supports describing a more comprehensive contextual image features. In this work, we define the texture features based on the GLCM of an image, which include the following: autocorrelation, contrast, correlation, cluster prominence, cluster shade, dissimilarity, energy, entropy, homogeneity, maximum probability, sum of squares: variance, sum average, sum variance, sum entropy, difference variance, difference entropy, information measure of correlation1, information measure of correlation2, inverse difference normalized, and inverse difference moment normalized.

\subsection{Parameter learning for SSTS}

There are three parameters that need to be set by users in SSTS, which are normalization threshold $m$ for SLIC over-segmentation, distance threshold $d$ for clustering super-pixels to form patches based on DBSCAN, and $r$ for patch labeling (see Fig. 5). Values of $m$ and $d$ determine the clustering performance of SLIC and DBSCAN, and hence influence the extent of the segmented tumor patches fitting to the ground truth. Therefore, it is vital to carefully set values of $m$ and $d$. We adopt an exhaustive

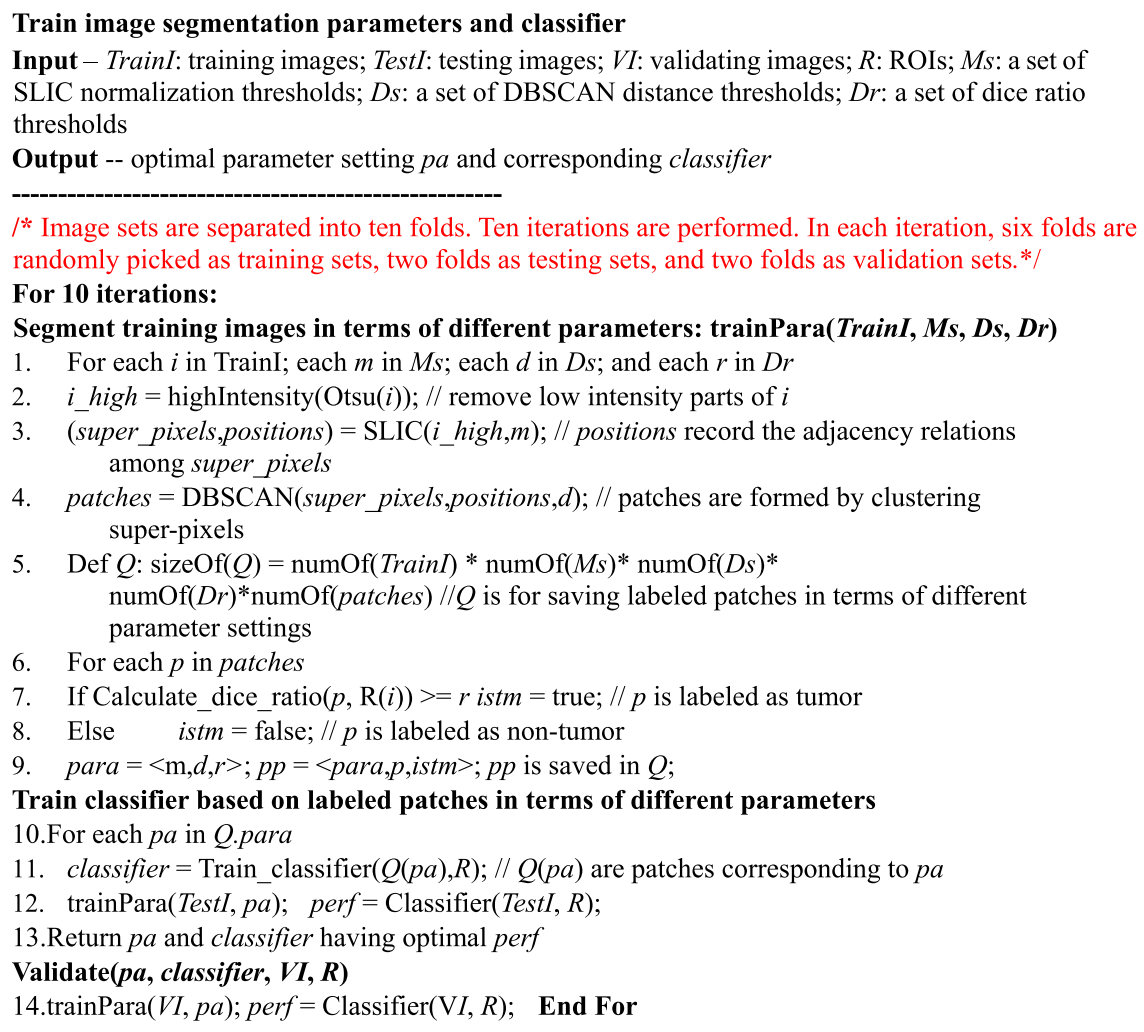

Fig. 5. Determination of optimal parameters for segmenting and classifying tumor patches. 
method based on extensive experiments to observe the performance in terms of pre-defined values of $m$ and $d$. As the performance of tumor extraction is also based on the patch classification results (i.e., whether patches are grouped to the right categories), training an efficient classifier plays a key role. Correctly labeling the segmented patches (tumor or nontumor) is the base of the classifier training. Threshold $r$ is defined for patch labeling.

The parameter training procedure is shown in Fig. 5. We separate the overall MRIs to three sets: training, testing and validating. Given an MRI $i$, if the DR between one patch $p$ of $i$ and the ground truth of $i(R(i))$ is not less than $r$, then $p$ is labeled as tumor (see lines 7-8 in Fig. 5). Given different values of $m$ and $d$, the patches of segmenting $i$ are different. And given different $r$, the labeling results of patches are different. Therefore, for each patch in a segmentation, we save this patch and its corresponding parameters $(m, d$ and $r$ ) in $Q$ (line 9 ). Each setting $\langle m, d, r\rangle$ corresponds to a set of labeled patches obtained from segmenting different MRIs. For each setting, we train a classifier based on its labeled patches (line 11). We validate the performance of the classifier based on the labeled patches of testI (line 12). At last, we use the validation set $V I$ to validate the trained parameters $p a$ and its corresponding classifier (line 14).

\section{Experiments}

Our experiments are conducted on Windows 10 $(\times 64)$, with Intel Core i7-6700HQ CPU, 2.6 GHZ and $16 \mathrm{~GB}$ RAM. We validate the SSTS method based on real and precisely labeled image sets, which are introduced in Sec. 4.1. We apply a 10-fold cross-validation technique (see Fig. 5): we separate image sets into 10 folds, and perform 10 iterations of training, testing and validating. In each iteration, we randomly pick six folds as training datasets, two folds as testing datasets, and two folds as validation datasets. We collected and labeled datasets in US, performed the experiments in both China and Australia, and finished the experiments in October, 2017.

\subsection{Dataset description}

We validate the proposed semi-supervised image segmentation method by using MRI image sets of 21 cases. Each case has a set of three-dimensional MRI images that are all in uint8 format. The gray-level range of MRIs is $0-255$. The size of each MRI is $512 \times 512 \times 162$ pixels. MRIs of 14 cases contain mass malignant lesions, and 7 have nonmass tumors, and 14 cases have infiltrating ductal carcinoma (IDC) tumor, 2 have the ductal carcinoma in situ (DCIS) of the breast, and 5 have both IDC and DCIS tumors.

Our experiment is mainly based on MRIs containing tumors whose shortest radius are over 17 pixels. Figure 7 shows two examples of tumors with short radius less than 17 pixels (i.e., very small tumors). The performance of SSTS on very small tumors is not as that efficient. The main reason is that the features of mean intensity values and textures of very small tumors cannot be described efficiently, so it is difficult to distinguish the very small tumor area with the other parts in an MRI. How to efficiently extract very small tumors of MRIs will be our future work.

\subsection{Classification validation of SSTS}

After the DBSCAN step of separating an MRI image into a set of patches, one tumor will be separated into different patches. SSTS then performs the classification step to identify patches of tumors, and combines the detected tumor patches to obtain a whole tumor. We first show the classification validation in this section.

From Fig. 1, the submodule label of Module 4 is to label the segmented patches of MRI, i.e., to determine whether a patch is tumor or nontumor. This labeling result is based on the segmentation results of Modules 1-3.

For each MRI layer containing tumors, we set a series of parameter values to train corresponding tumor extraction models (inputs in Fig. 5), which are $M s=\langle 5,10,15,20,25\rangle, \quad D s=\langle 3,5,8,10,15\rangle$, and $D r=\langle 0.1,0.2, \ldots, 0.9\rangle$. For each setting of parameters, we train one classifier based on the segmented patches. Each parameter setting corresponds to around 500 tumor patches and 4000 nontumor patches. To resolve the problem of data unbalancing, we use SMOTE method ${ }^{26}$ to generate synthetic tumor samples, and use under-sampling method to reduce the nontumor patches. Overall, we have 225 classifiers with respect to different parameter combinations. The trained classifiers can 


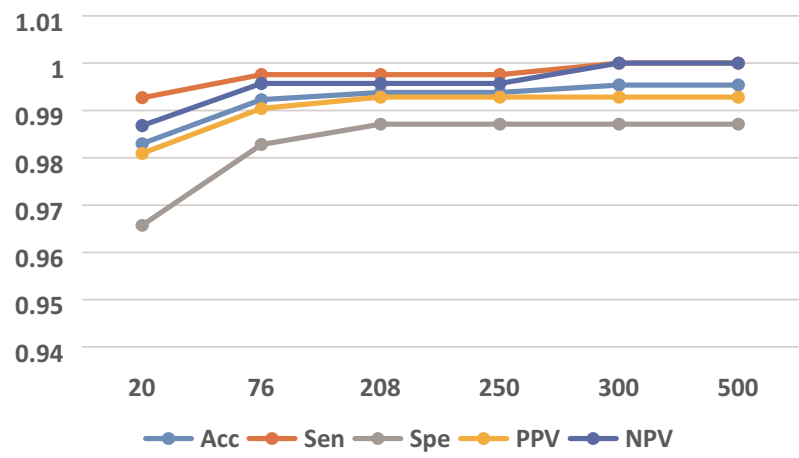

(a)

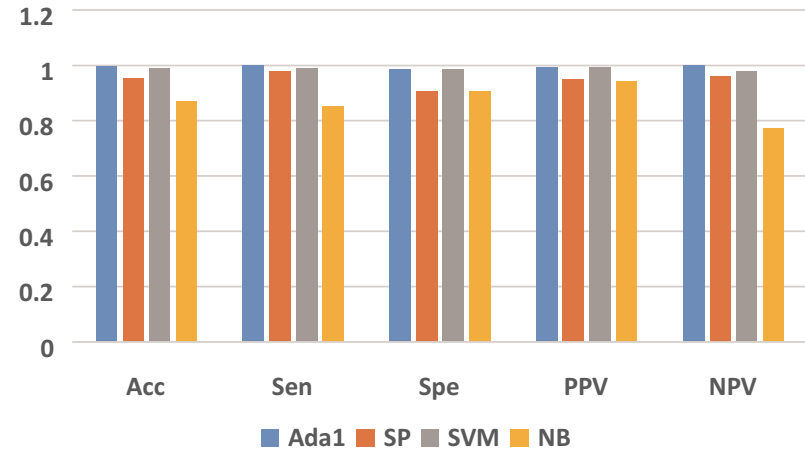

(b)

Fig. 6. Classification performance validation. (a) Performance comparison in terms of different tree numbers and (b) performance comparison of four classifiers.
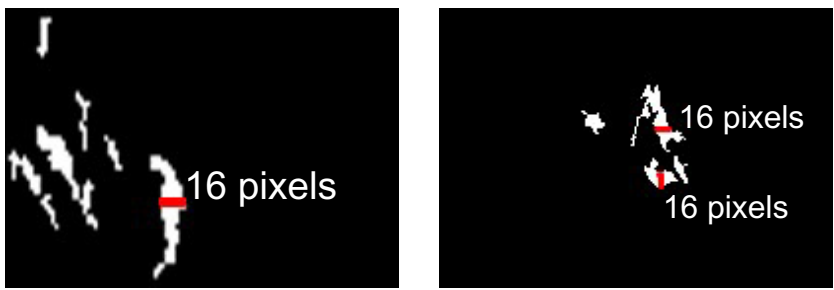

Fig. 7. Two examples of very small tumors.

also be used for detecting which breast (right or left) contains tumors in an MRI.

Based on the empirical validation results with respect to the predefined values of $M s, D s$ and $D r$, we fix $\langle m, d, r\rangle=\langle 15,8,0.6\rangle$ (Modules 2-4) for segmenting trainI, testI and VI, and labeling segmented patches as tumors or nontumors. Figure 6 shows the classification performance based on the textures and mean intensity levels of image patches with respect to $\langle m, d, r\rangle=\langle 15,8,0.6\rangle$. We present the performance of Adaboost-M1 algorithm in terms of different decision tree numbers $(20,76,208$, 250, 300, and 500) (see Fig. 6(a)), where the optimal number of trees is 300 . We then fix the tree number as 300, and compare the performance of Adaboostm1 (Ada1) with another three classification algorithms: random subspace (SP) ensemble algorithm, ${ }^{13}$ Support Vector Machine (SVM), ${ }^{27}$ and Naive Bayes $(\mathrm{NB})^{28}$ (see Fig. 6(b)). We can see that Adaboost-M1 with 300 decision trees has optimal performance compared with the other three algorithms.

\subsection{Qualitative validation of tumor extraction of SSTS}

For both mass and nonmass tumors, we qualitatively compare and show the segmentation results of SSTS with the performance of an MT method, ${ }^{10}$ and an FCM method. ${ }^{14}$ MT uses Otsu's multi-level thresholding method to segment images. We then pick up and combine the unconnected tumor patches in a segmented image, and then validate the performance of MT and FCM based on the extracted tumor patches.

The parameters of FCM and MT that need to be predefined is the cluster number (cn) and the level number (ln) of clustering pixels of an MRI. We use similar parameter setting method of SSTS to set cn and ln: after segmenting an MRI into small patches, we label each patch based on a fixed DR value (between a patch and the ROI), and learn AdabootM1 classifier (with 300 simple trees) based on the labeled patches. The parameters resulting in the optimal DR (between an extracted tumor area and the ROI) are fixed to validate the performance of FCM and MT based on VI. The optimal parameters are $\langle\mathrm{cn}, d r\rangle=\langle 3,0.4\rangle$ and $\langle\ln , d r\rangle=\langle 5,0.5\rangle$ (see Table 2).

After fixing parameters of SSTS, MT, and FCM, we compare the tumor extraction performance of SSTS, MT and FCM on mass and non-mass tumors. Figure 8 shows the extraction results of mass tumors. We can see that the extraction results of SSTS on MRIs of case 1 are similar as the result of MT, which is much better than the result of FCM. The results of SSTS on MRIs of cases 3-6 are similar as the results of FCM, which are better than the results of MT. The segmentation results of SSTS on MRIs of cases 6-9 are significantly better than the results of MT and FCM. Figure 9 shows seven examples of extracting results of non-mass tumors. We can see that SSTS performs much better on extracting 


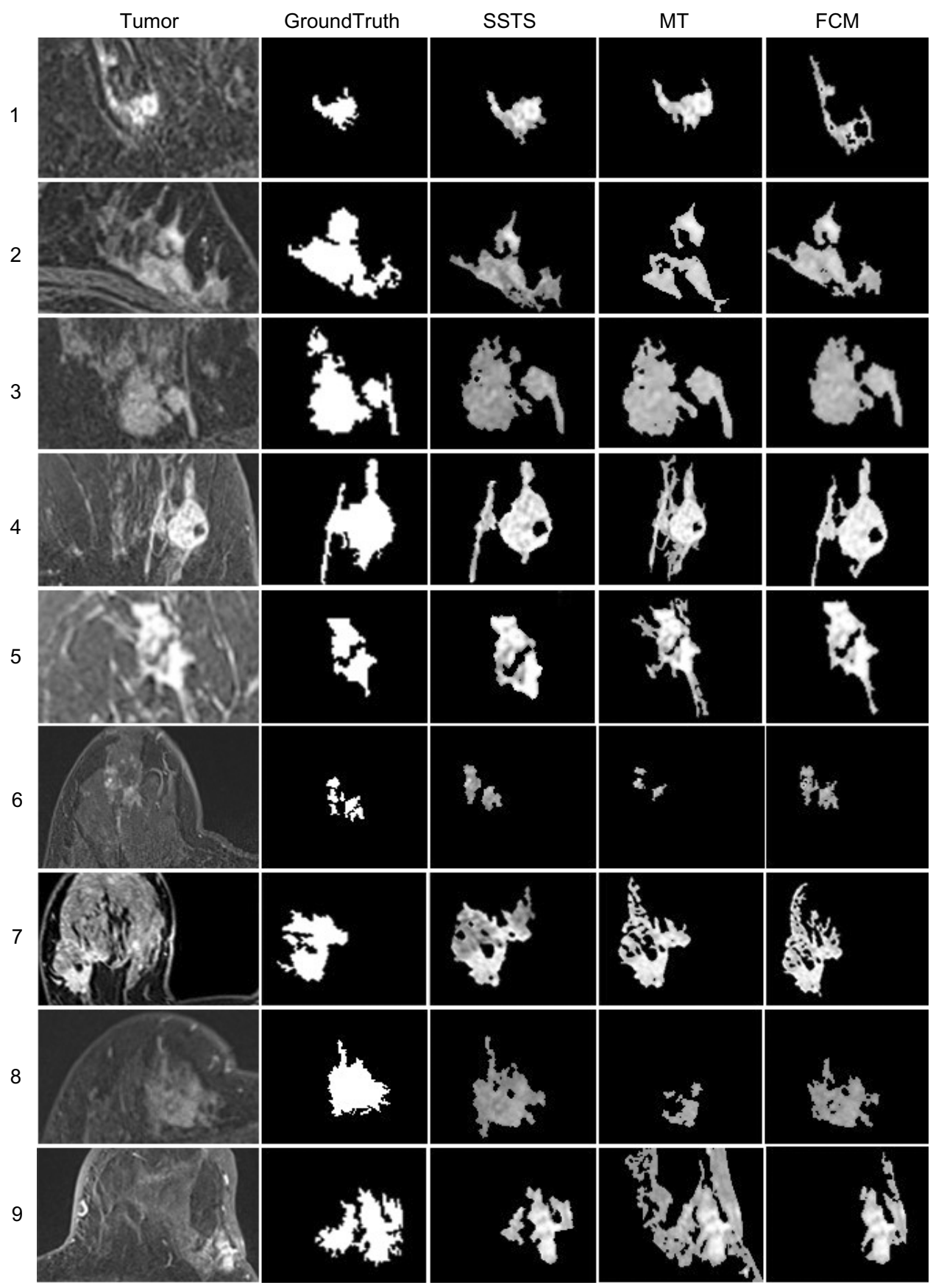

Fig. 8. Comparison of segmentation results of SSTS, MT, and FCM on mass tumors of nine cases.

non-mass tumors compared with the MT and FCM methods.

\subsection{Quantitative validation of tumor extraction of SSTS}

We use a set of metrics to quantitatively validate the performance of different segmentation methods. Definitions of metrics are defined in Table 1, including DR, Precision (PR) and Recall (RC). $S$ represents the segmented area and $G$ denotes the ground truth.

We quantitatively compare the tumor extraction performance of SSTS, FCM, MT, Statistical moment (SM)-based $k$-means clustering, ${ }^{29}$ and Morphology (MOR)-based $k$-means clustering. ${ }^{30}$ The parameter settings of SSTS, FCM, and MT were discussed in Sec. 4.3. 
Table 1. Definitions of validation metrics.

\begin{tabular}{lc}
\hline Metrics & Formulas \\
\hline $\mathrm{DR}$ & $\frac{2(S \cap G)}{S \cap G+S \cup G} \cdot 100$ \\
$\mathrm{PR}$ & $S \cap G / S$ \\
$\mathrm{RC}$ & $S \cap G / G$ \\
\hline
\end{tabular}
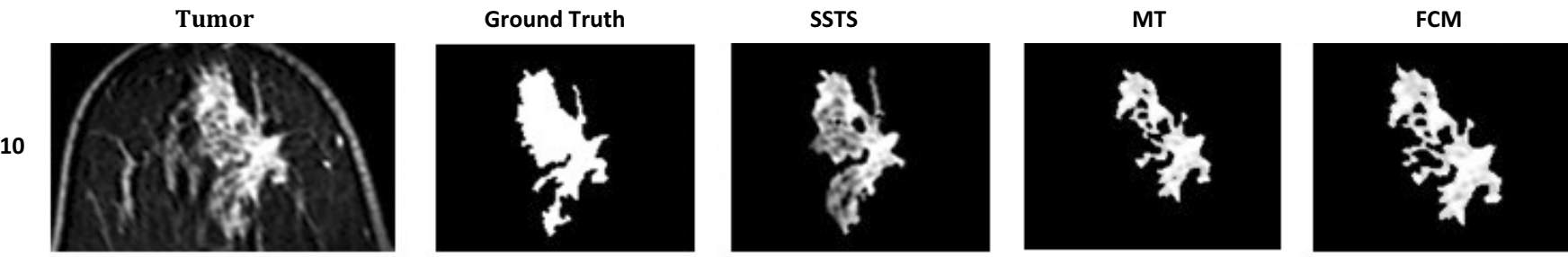

11
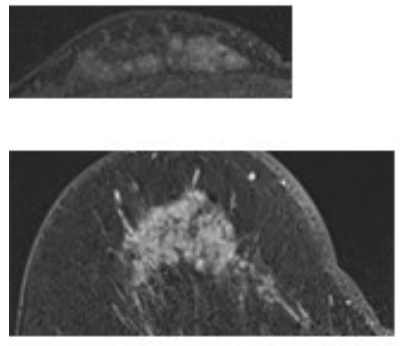

13
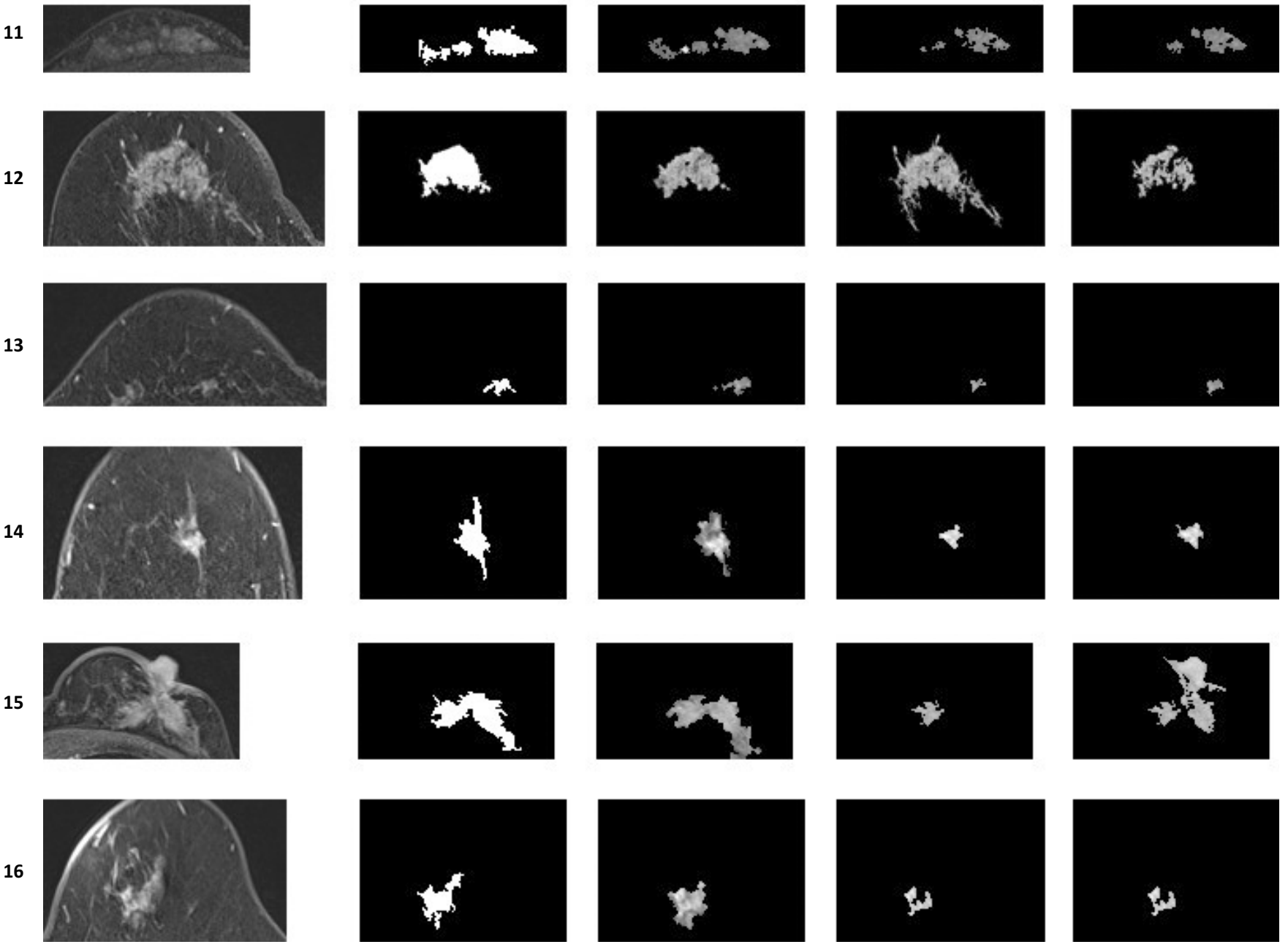

Fig. 9. Comparison of segmentation results of SSTS, MT, and FCM on nonmass tumors of seven cases. 


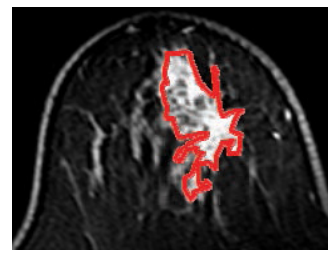

(a) Ground Truth

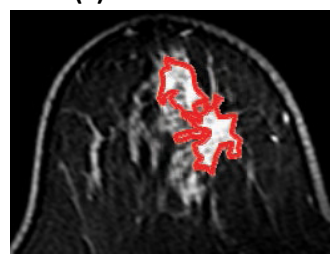

(c) MT

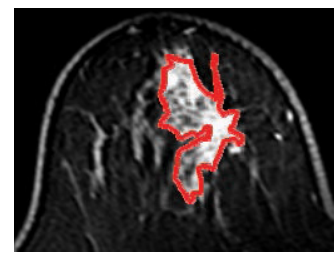

(b) SSTS

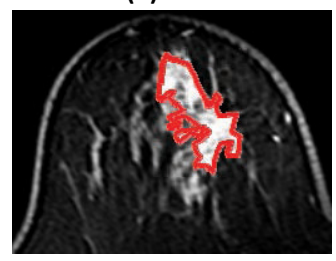

(d) FCM

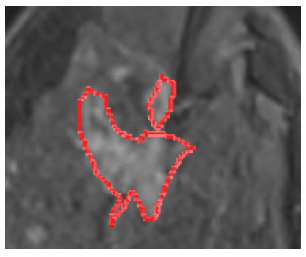

(a) Ground Truth

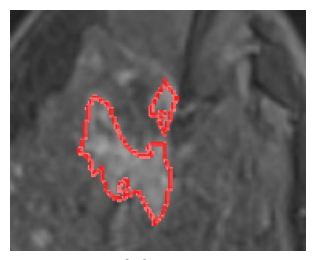

(c) MT

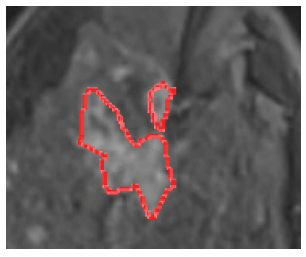

(b) SSTS

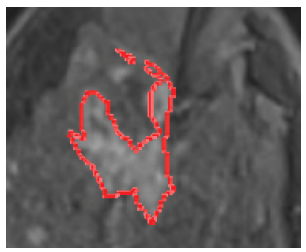

(d) FCM

(a) Segmentation results of case 10

(b) Segmentation results of case 6

Fig. 10. Zoom in the segmented tumors of ground truth, SSTS, MT, and FCM.

For SM method, we use sliding windows to separate each image into small patches and process each patch step-by-step. We fix the sliding window size as 41, and the step size of sliding window as 1 . Each image patch is calculated to obtain a feature vector that contains five first statistical moments, and all the sub-images are clustered in terms of their feature vectors. The MOR method applied morphological filtering and reconstruction schema to segment circular regions in an image. We fix the segmentation sensitivity as 0.02 , and the kernel size ranged from 5 to 20 (see Table 2).

From Table 2, the DR of SSTS is better than the DR of the others, while the precision of SSTS is worse than the precision of FCM and MT. Based on the definition of DR, PR, and RC (Table 1), the delineated area of SSTS tends to be larger than the true ground tumor area (ROI), while the FCM and MT tend to delineate an ROI's sub-area that cannot cover the whole tumor. The SM and MOR perform dramatically worst compared with the other three methods (based on DR), even though their recalls are better than the others, which indicates that the $\mathrm{SM}$ and MOR extract regions are much larger than the true ROI area.

Our experiment is mainly based on MRIs containing tumors whose shortest radius are over 17 pixels. Figure 10 shows two examples of tumors with short radius less than 17 pixels (namely very small tumors). The performance of SSTS based on very small tumors is not as efficient as its performance on normal tumors. The main reason is that the features textures of very small tumors cannot be described as well as the features of normal tumors, so it is difficult to distinguish the very small tumors area with the other parts in an MRI. How to efficiently extract very small tumors of MRIs will be our future work.

\section{Conclusion and Future Work}

This work developed an SSTS method to delineate lesions in breast MRIs. The proposed method requires limited number of parameters to be set in advance, and is capable of delineating both mass and nonmass tumors. In SSTS, we applied Otsu thresholding method to remove low intensity pixels, and used SLIC super-pixel method to over-segment MRIs, both of which aim to improve the efficiency of tumor segmentation. We validate SSTS on a large amount of labeled MR images, and the experimental results show higher DR of SSTS on extracting both mass and nonmass tumors in breast MRIs compared with classical image segmentation methods (e.g., MT and FCM). SSTS can also be extended to distinguish health and cancerous breasts based on MRIs. However, the current parameter setting process is an exhaustive procedure, and only discrete parameter values are tested. Our future work will be on developing a more efficient parameter setting procedure; and extend our work to extracting more types of breast tumors and very small tumors.

\section{Conflict of Interest}

The authors declare that there are no conflicts of interest related to this paper. 


\section{Acknowledgement}

This paper is supported by the National Natural Science Foundation of China (Grants No 61702274) and the Natural Science Foundation of Jiangsu Province (Grants No BK20170958).

\section{References}

1. P. Ghamisi, M. S. Couceiro, F. M. Martins, J. A. Benediktsson, "Multilevel image segmentation based on fractional-order Darwinian particle swarm optimization," IEEE Trans. Geosci. Remote Sens. 52(5), 2382-2394 (2014).

2. D. L. Pham, C. Xu, J. L. Prince, "Current methods in medical image segmentation," Annu. Rev. Biomed. Eng. 2(1), 315-337 (2000).

3. J. Ma, A. Jemal, Breast cancer statistics, Breast Cancer Metastasis and Drug Resistance, pp. 1-18, Springer, New York (2013).

4. W. Chen, M. L. Giger, U. Bick, "A fuzzy c-means (FCM)-based approach for computerized segmentation of breast lesions in dynamic contrast-enhanced MR images," Acad. Radiol. 13(1), 63-72 (2006).

5. N. Gordillo, E. Montseny, P. Sobrevilla, "State-ofthe-art survey on MRI brain tumor segmentation," Magn. Reson. Imag. 31(8), 1426-1438 (2013).

6. A. B. Ashraf, S. C. Gavenonis, D. Daye, C. Mies, M. A. Rosen, D. Kontos, "A multichannel Markov random field framework for tumor segmentation with an application to classification of gene expression-based breast cancer recurrence risk," IEEE Trans. Med. Imag. 32(4), 637-648 (2013).

7. G. P. Liney, M. Sreenivas, P. Gibbs, R. GarciaAlvarez, L. W. Turnbull, "Breast lesion analysis of shape technique: Semiautomated vs. manual morphological description," J. Magn. Reson. Imag. 23(4), 493-498 (2006).

8. W. Chen, M. L. Giger, L. Lan, U. Bick, "Computerized interpretation of breast MRI: Investigation of enhancement-variance dynamics," Med. Phys. 31(5), 1076-1082 (2004).

9. K. Nie, J. H. Chen, J. Y. Hon, Y. Chu, O. Nalcioglu, M. Y. Su, "Quantitative analysis of lesion morphology and texture features for diagnostic prediction in breast MRI," Acad. Radiol. 15(12), 15131525 (2008).

10. N. Gordillo, E. Montseny, P. Sobrevilla, "State-ofthe-art survey on MRI brain tumor segmentation," Magn. Reson. Imag. 31(8), 1426-1438 (2013).

11. N. Otsu, "A threshold selection method from graylevel histograms," IEEE Trans. Syst. Man Cybern. $\mathbf{9}(1), 62-66$ (1979).
12. R. Achanta, A. Shaji, K. Smith, A. Lucchi, P. Fua, S. Süsstrunk, "SLIC superpixels compared to state-of-the-art superpixel methods," IEEE Trans. Pattern Anal. Mach. Intell. 34(11), 2274-2282 (2012).

13. J. Shen, X. Hao, Z. Liang, Y. Liu, W. Wang, L. Shao, "Real-time superpixel segmentation by DBSCAN clustering algorithm," IEEE Trans. Image Process. 25(12), 5933-5942 (2016).

14. S. Kotsiantis, "Combining bagging, boosting, rotation forest and random subspace methods," Artif. Intell. Rev. 35(3), 223-240 (2011).

15. J. Li, X. Li, B. Yang, X. Sun, Segmentation-based image copy move forgery detection scheme, IEEE Transactions on Information Forensics and Security 10(3), 507-518 (2015).

16. M. Gong, Y. Liang, J. Shi, W. Ma, J. Ma, "Fuzzy c-means clustering with local information and kernel metric for image segmentation," IEEE Trans. Image Process. 22(2), 573-584 (2013).

17. M. Havaei, A. Davy, D. Warde-Farley, A. Biard, A. Courville, Y. Bengio, C. Pal, P. M. Jodoin, H. Larochelle, "Brain tumor segmentation with deep neural networks," Med. Image Anal. 35, 18-31 (2017).

18. N. Sauwen, M. Acou, S. Van Cauter, D. M. Sima, J. Veraart, F. Maes, U. Himmelreich, E. Achten, S. Van Huffel, "Comparison of unsupervised classification methods for brain tumor segmentation using multi-parametric MRI," NeuroImage 12, 753-764 (2016).

19. S. Pereira, A. Pinto, V. Alves, C. A. Silva, "Brain tumor segmentation using convolutional neural networks in MRI images," IEEE Trans. Med. Imag. 35(5), 1240-1251 (2016).

20. A. Sehgal, S. Goel, P. Mangipudi, A. Mehra, D. Tyagi, Automatic brain tumor segmentation and extraction in MR images, Conf. Advances in Signal Processing (CASP), pp. 104-107, IEEE, Pune, India, (2016).

21. A. Q. Al-Faris, U. K. Ngah, N. A. M. Isa, I. L. Shuaib, Breast MRI tumor segmentation using modified automatic seeded region growing based on particle swarm optimization image clustering, Soft Computing in Industrial Applications, pp. 49-60, Springer, Cham (2014).

22. X. X. Yin, S. Hadjiloucas, J. H. Chen, Y. Zhang, J. L. Wu, M. Y. Su, "Correction: Tensor-based multichannel reconstruction for breast tumors identification from DCE-MRIs," PLoS One 12(4), e0176133 (2017).

23. T. Pevny, P. Bas, J. Fridrich, "Steganalysis by subtractive pixel adjacency matrix," IEEE Trans. Inf. Forensics Sec. 5(2), 215-224 (2014).

24. P. Koversi, Image segmentation using SLIC superpixels and DBSCAN clustering (2014), http://www. 
peterkovesi.com/projects/segmentation/. Accessed on September 2017.

25. Y. Freund, R. E. Schapire, Experiments with a new boosting algorithm, In Proc. Thirteenth Int. Conf. Machine Learning, pp. 148-156, San Francisco, Morgan Kaufmann, (1996).

26. R. M. Haralick, K. Shanmugam, Textural features for image classification, IEEE Trans. Syst. Man Cybern. SMC-3(6), 610-621 (1973).

27. L. K. Soh, C. Tsatsoulis, "Texture analysis of SAR sea ice imagery using gray level co-occurrence matrices," IEEE Trans. Geosci. Remote Sens. 37(2), 780-795 (1999).

28. N. V. Chawla, K. W. Bowyer, L. O. Hall et al., "SMOTE: Synthetic minority over-sampling technique," J. Artif. Intell. Res. 16, 321-357 (2002).

29. J. A. K. Suykens, J. Vandewalle, "Least squares support vector machine classifiers," Neural Process. Lett. 9(3), 293-300 (1999).
30. L. Rish, An empirical study of the naive bayes classifier, IJCAI 2001 Workshop on Empirical Methods in Artificial Intelligence, pp. 41-46, IBM, New York (2001).

31. M. K. Hu, "Visual pattern recognition by moment invariants," IRE Trans. Inf. Theory 8(2), 179-187 (1962).

32. B. Irving, P. Taylor, A. Todd-Pokropek, 3D segmentation of the airway tree using a morphology based method, Proc. of Second Int. Workshop on Pulmonary Image Analysis, pp. 297-307 (2009).

33. A. Usinskas, B. Tomandl, P. Hastreiter, K. Spinnler, T. Wittenberg, Improvements on the gray level cooccurrence matrix technique to compute ischemic stroke volume, Bildverarbeitung für die Medizin, pp. 267-270 (2002). 